\title{
Analyzing the Limitations of Cross-lingual Word Embedding Mappings
}

\author{
Aitor Ormazabal, Mikel Artetxe, Gorka Labaka, Aitor Soroa, Eneko Agirre \\ IXA NLP Group \\ University of the Basque Country (UPV/EHU) \\ aormazabal024@ikasle. ehu. eus \\ \{mikel.artetxe, gorka.labaka, a.soroa, e.agirre\}dehu.eus
}

\begin{abstract}
Recent research in cross-lingual word embeddings has almost exclusively focused on offline methods, which independently train word embeddings in different languages and map them to a shared space through linear transformations. While several authors have questioned the underlying isomorphism assumption, which states that word embeddings in different languages have approximately the same structure, it is not clear whether this is an inherent limitation of mapping approaches or a more general issue when learning crosslingual embeddings. So as to answer this question, we experiment with parallel corpora, which allows us to compare offline mapping to an extension of skip-gram that jointly learns both embedding spaces. We observe that, under these ideal conditions, joint learning yields to more isomorphic embeddings, is less sensitive to hubness, and obtains stronger results in bilingual lexicon induction. We thus conclude that current mapping methods do have strong limitations, calling for further research to jointly learn cross-lingual embeddings with a weaker cross-lingual signal.
\end{abstract}

\section{Introduction}

Cross-lingual word embeddings have attracted a lot of attention in recent times. Existing methods can be broadly classified into two categories: joint methods, which simultaneously learn word representations for multiple languages on parallel corpora (Gouws et al., 2015; Luong et al., 2015), and mapping methods, which independently train word embeddings in different languages and map them to a shared space through linear transformations (Mikolov et al., 2013a; Artetxe et al., 2018a). While early work in cross-lingual word embeddings was dominated by joint approaches, recent research has almost exclusively focused on mapping methods, which have the advantage of requir- ing little or no cross-lingual signal (Zhang et al., 2017; Conneau et al., 2018; Artetxe et al., 2018b).

For mapping methods to work, it is necessary that embedding spaces in different languages have a similar structure (i.e. are approximately isomorphic), as it would otherwise be hopeless to find a linear map from one space to another. Several authors have questioned this assumption, showing that linguistic and domain divergences cause strong mismatches in embedding spaces, which in turn heavily hinders the performance of these methods (Søgaard et al., 2018; Patra et al., 2019). Nevertheless, it is not clear whether this mismatch is a consequence of separately training both embedding spaces, and thus an inherent limitation of mapping approaches, or an insurmountable obstacle that arises from the linguistic divergences across languages, and hence a more general issue when learning cross-lingual word embeddings.

The goal of this paper is to shed light on this matter so as to better understand the nature and extension of these limitations. For that purpose, we experiment with parallel corpora, which allows us to compare mapping methods and joint methods under the exact same conditions, and analyze the properties of the resulting embeddings. Our results show that, under these conditions, joint learning yields to more isomorphic embeddings, is less sensitive to hubness, and obtains stronger results in Bilingual Lexicon Induction (BLI). This suggests that, despite the advantage of requiring weaker cross-lingual signal, current mapping methods do have strong limitations, as they are not able to leverage the available evidence as effectively as joint methods under ideal conditions. We thus conclude that future research should try to combine the best of both worlds, exploring joint methods to learn cross-lingual word embeddings with weaker supervision. 


\section{Related work}

Cross-lingual word embeddings represent words from multiple languages in a common vector space. So as to train them, joint methods simultaneously learn the embeddings in the different languages, which requires some form of cross-lingual supervision. This supervision usually comes from parallel corpora, which can be aligned at the word level (Luong et al., 2015), or only at the sentence level (Gouws et al., 2015). In addition to that, methods that rely on comparable corpora (Vulić and Moens, 2016) or large bilingual dictionaries (Duong et al., 2016) have also been proposed. For a more detailed survey, the reader is referred to Ruder et al. (2017).

In contrast, offline mapping approaches work by aligning separately trained word embeddings in different languages. For that purpose, early methods required a training dictionary, which was used to learn a linear transformation that mapped these embeddings into a common space (Mikolov et al., 2013a; Artetxe et al., 2018a). The amount of required supervision was later reduced through selflearning methods (Artetxe et al., 2017), and then completely eliminated through adversarial training (Zhang et al., 2017; Conneau et al., 2018) or more robust iterative approaches combined with initialization heuristics (Artetxe et al., 2018b; Hoshen and Wolf, 2018).

There are several authors that have discussed the potential limitations of these mapping approaches. For instance, Søgaard et al. (2018) observe that the assumption that separately trained embeddings are approximately isomorphic is not true in general, showing that the performance of mapping methods is conditioned by the language pair, the comparability of the training corpora, and the parameters of the word embedding algorithms. Similarly, Patra et al. (2019) show that the isomorphism assumption weakens as the languages involved become increasingly etymologically distant. Finally, Nakashole and Flauger (2018) argue that embedding spaces in different languages are linearly equivalent only at local regions, but their global structure is different. Nevertheless, neither of these works does systematically analyze the extent to which these limitations are inherent to mapping approaches. To the best of our knowledge, ours is the first work comparing joint and mapping methods in the exact same conditions, characterizing the nature and impact of such limitations.

\section{Experimental design}

We next describe the cross-lingual embedding methods, evaluation measures and datasets used in our experiments.

\subsection{Cross-lingual embedding methods}

We use the following procedure to learn crosslingual embeddings, which are representative of the state-of-the-art in mapping and joint methods: Mapping: We first train 300-dimensional skipgram embeddings for each language using word2vec (Mikolov et al., 2013b) with 10 negative samples, a sub-sampling threshold of 1e-5 and 5 training iterations. Having done that, we map the resulting monolingual embeddings to a cross-lingual space using the unsupervised mode in VecMap ${ }^{1}$ (Artetxe et al., 2018b), which builds an initial solution based on heuristics and iteratively improves it through self-learning.

Joint learning: We use the $\mathrm{BiVec}^{2}$ tool proposed by Luong et al. (2015), an extension of skip-gram that, given a word aligned parallel corpus, learns to predict the context of both the source word and the target word aligned with it. For that purpose, we first word align our training corpus using FastText (Dyer et al., 2013). Given that BiVec is a natural extension of skip-gram, we use the exact same hyperparameters as for the mapping method.

In both cases, we restrict the vocabulary to the most frequent 200,000 words.

\subsection{Evaluation measures}

We use the following measures to characterize cross-lingual embeddings:

Isomorphism. Intuitively, the notion of isomorphism captures the idea of how well the embeddings in both languages fit together (i.e. the degree of their structural similarity). So as to measure it, we use the eigenvalue similarity metric proposed by Søgaard et al. (2018). For that purpose, we first center and normalize the embeddings, calculate the nearest neighbor graphs of the 10,000 most frequent words in each language, and compute their Laplacian matrices $L_{1}$ and $L_{2}$. We then find the smallest $k_{1}$ such that the sum of the largest $k_{1}$ eigenvalues of $L_{1}$ is at least $90 \%$ of the sum of all its eigenvalues, and analogously for $k_{2}$ and $L_{2}$. Finally we set $k=\min \left(k_{1}, k_{2}\right)$, and define the eigenvalue similarity of the two spaces as the sum

\footnotetext{
${ }^{1}$ https://github.com/artetxem/vecmap

${ }^{2}$ https://github.com/lmthang/bivec
} 


\begin{tabular}{|c|c|c|c|c|c|c|c|c|c|c|}
\hline & & \multirow{2}{*}{$\begin{array}{c}\text { Eig. } \\
\text { sim. }(\downarrow)\end{array}$} & \multicolumn{2}{|c|}{ Hub. NN ( $\uparrow)$} & \multicolumn{2}{|c|}{ Hub. CSLS ( $\uparrow)$} & \multicolumn{2}{|c|}{ P@ 1 Eparl ( $\uparrow)$} & \multicolumn{2}{|c|}{ P@ 1 MUSE ( $\uparrow)$} \\
\hline & & & $10 \%$ & $100 \%$ & $10 \%$ & $100 \%$ & $\mathrm{NN}$ & CSLS & $\mathrm{NN}$ & CSLS \\
\hline \multirow{2}{*}{ FI-EN } & Joint learning & 28.9 & 0.45 & 52.8 & 1.13 & 57.5 & 65.2 & 68.3 & 83.4 & 85.2 \\
\hline & Mapping & 115.9 & 0.12 & 33.8 & 0.38 & 46.1 & 26.3 & 34.8 & 44.6 & 56.8 \\
\hline \multirow{2}{*}{ ES-EN } & Joint learning & 31.2 & 0.65 & 66.0 & 1.40 & 71.3 & 68.7 & 69.3 & 91.9 & 92.4 \\
\hline & Mapping & 47.8 & 0.58 & 63.1 & 1.31 & 69.1 & 65.4 & 67.0 & 87.1 & 89.0 \\
\hline \multirow{2}{*}{ DE-EN } & Joint learning & 32.8 & 0.58 & 58.8 & 1.29 & 65.2 & 70.6 & 70.4 & 90.1 & 89.2 \\
\hline & Mapping & 39.4 & 0.60 & 58.7 & 1.33 & 64.8 & 65.3 & 66.4 & 82.4 & 83.1 \\
\hline \multirow{2}{*}{ IT-EN } & Joint learning & 26.5 & 0.75 & 69.7 & 1.61 & 74.2 & 71.5 & 71.8 & 90.6 & 90.0 \\
\hline & Mapping & 43.9 & 0.65 & 63.9 & 1.53 & 70.8 & 64.1 & 67.2 & 84.4 & 85.9 \\
\hline
\end{tabular}

Table 1: Evaluation measures for the two cross-lingual embedding approaches. Arrows indicate whether lower $(\downarrow)$ or higher $(\uparrow)$ is better. See text for further details.

of the squared differences between the $k$ largest eigenvalues of $L_{1}$ and $L_{2}, \Delta=\sum_{i=1}^{k}\left(\lambda_{1_{i}}-\lambda_{2_{i}}\right)^{2}$.

Hubness. Cross-lingual word embeddings are known to suffer from the hubness problem (Radovanović et al., 2010a,b; Dinu et al., 2015), which causes a few points (known as hubs) to be the nearest neighbors of many other points in high-dimensional spaces. So as to quantify it, we measure the minimum percentage of target words $H_{N}$ that are the nearest neighbor of at least $N \%$ of the source words, where $N$ is a parameter of the metric. ${ }^{3}$ For instance, a hubness value of $H_{10 \%}=0.3 \%$ would indicate that $0.3 \%$ of the target words are the nearest neighbors of $10 \%$ of the source words. This way, lower values of $H_{N}$ are indicative of a higher level of hubness, and the parameter $N$ serves to get a more complete picture of the distribution of hubs. For brevity, we report results for $N=10 \%$ and $100 \%$. While the nearest neighbor retrieval is usually done according to cosine similarity, Conneau et al. (2018) proposed an alternative measure, called Cross-domain Similarity Local Scaling (CSLS), that penalizes the similarity scores of hubs, which in turn reduces the hubness level. So as to better understand its effect, we report results for both CSLS and standard nearest neighbor with cosine similarity $(\mathrm{NN})$.

\footnotetext{
${ }^{3}$ Some previous work uses an alternative hubness metric that computes the hubness level $N(t)$ of each target word $t$ (i.e. the number of source words whose nearest neighbor is $t$ ) and measures the skewness of the resulting distribution. However, we find this metric to have two important drawbacks: 1) its magnitude is not easily interpretable, and 2) it is invariant to the variance of the distribution, even if higher variances are indicative of a higher hubness level. For instance, we observed that two very similar spaces (produced running word2vec twice over the same corpora) mapped to each other produced unusually high skewness scores, caused by the scale normalization done in skewness (division by the standard deviation).
}

Bilingual Lexicon Induction (BLI). Following common practice, we induce a bilingual dictionary by linking each word in the source language with its nearest neighbor in the target language. So as to evaluate the quality of the induced translations, we compare them to a gold standard dictionary, and measure the precision at 1 . We report results for both nearest neighbor with cosine similarity (NN) and the aforementioned CSLS retrieval. Note that, in addition to having a practical application, BLI performance is an informative measure of the quality of the embeddings, as a good cross-lingual representation should place equivalent words close to each other.

\subsection{Datasets}

We experiment with 4 language pairs with English as the target language, covering 3 relatively close languages (German, Spanish and Italian) and a non-indoeuropean agglutinative language (Finnish). All embeddings were trained on the BiCleaner v3.0 version of the ParaCrawl corpus, ${ }^{4}$ a parallel corpus collected through crawling and filtered according to Sánchez-Cartagena et al. (2018). The size of this corpus changes from one language to another: German and Spanish are the largest (503 and 492 million tokens in the English side, respectively), followed by Italian (308 million tokens), and Finnish (55 million tokens).

As for the evaluation dictionaries for BLI, we use two datasets that have been widely used in the literature. The first one, which we call Eparl, was first introduced by Dinu et al. (2015) and subsequently extended by Artetxe et al. (2017) and Artetxe et al. (2018a), and consists of 1,500 test entries extracted from Europarl word alignments

\footnotetext{
${ }^{4}$ https://paracrawl.eu/
} 
and uniformly distributed in 5 frequency bins. The second one, which we call MUSE, consists of another 1,500 test entries, and was compiled by Conneau et al. (2018) using internal translation tools.

\section{Results}

Table 1 reports the results of all the evaluation measures for both cross-lingual embedding approaches.

The eigenvalue similarity metric shows that joint learning obtains substantially more isomorphic embedding spaces than the mapping approach, indicating that the representations it learns for different languages have a more similar structure. At the same time, it is remarkable that the eigenvalue similarity for the four language pairs is very close in the case of joint learning, with values that range between 26.5 and 32.8. In contrast, the degree of isomorphism for Finnish-English is substantially lower than the rest in the case of the mapping approach, which is likely caused by the typological differences between these languages and the smaller size of the training corpus. This suggests that joint learning is able to appropriately fit divergent languages together, which is troublesome when the embedding spaces are learned separately and then mapped together.

When it comes to hubness, our results show that joint learning is generally less sensitive to this problem, although differences greatly vary depending on the language pair. This way, both approaches have a similar behavior in German, while joint learning does moderately better for Spanish and Italian, and the difference becomes very large for Finnish. Once again, this suggests that mapping methods are more severely affected by linguistic divergences. At the same time, we observe that CSLS is very effective at reducing the hubness level, specially for offline mapping.

Finally, we observe that joint learning outperforms offline mapping in BLI. This difference is particularly pronounced for Finnish-English (e.g. $26.3 \%$ vs $65.2 \%$ for $\mathrm{NN}$ on Eparl), which is in line with the general behavior observed so far. At the same time, our results show that CSLS is most helpful with offline mapping, but it even has a negative impact with joint learning for some language pairs. This can be partly explained by the fact that the latter approach is less sensitive to hubness, which CSLS tries to address.

\section{Discussion}

Our analysis reveals that, when trained on parallel corpora under the exact same conditions, joint learning obtains substantially better cross-lingual representations than offline mapping, yielding to more isomorphic embeddings that are less sensitive to hubness and obtain stronger results on BLI. Moreover, our results show that divergences across languages can be effectively mitigated by jointly learning their representations, whereas trying to align separately trained embeddings is troublesome when such divergences exist.

Note that this should not be interpreted as a claim that existing joint methods are superior to existing mapping methods. In fact, we believe that both families serve different purposes in that they require a different degree of supervision (e.g. mapping methods can exploit monolingual corpora, which is useful in practical settings), so the choice to use one approach or the other should depend on the resources that are available in each particular case. Nevertheless, our results do show that offline mapping has fundamental limitations that, given the available evidence, seem specific to this particular approach.

For that reason, we argue that, while recent research on cross-lingual word embeddings has almost exclusively focused on mapping methods, future work should consider alternative approaches to try to overcome the limitations of this paradigm. In particular, we believe that an interesting direction would be to adapt joint methods so they can work with monolingual corpora.

\section{Conclusions and future work}

In this work, we compare the properties of crosslingual word embeddings trained through joint learning and offline mapping on parallel corpora. We observe that, under these ideal conditions, joint learning yields to more isomorphic embeddings, is less sensitive to hubness, and obtains stronger results in bilingual lexicon induction, concluding that current mapping methods have strong limitations.

This analysis calls for further research on alternatives to current mapping methods, which have been very successful on unsupervised settings. In particular, we would like to explore new methods to jointly learn cross-lingual embeddings on monolingual corpora. 


\section{Acknowledgments}

This research was partially supported by the Spanish MINECO (UnsupNMT TIN2017-91692EXP and DOMINO PGC2018-102041-B-I00, cofunded by EU FEDER), the BigKnowledge project (BBVA foundation grant 2018), the UPV/EHU (excellence research group), and the NVIDIA GPU grant program. Mikel Artetxe was supported by a doctoral grant from the Spanish MECD.

\section{References}

Mikel Artetxe, Gorka Labaka, and Eneko Agirre. 2017. Learning bilingual word embeddings with (almost) no bilingual data. In Proceedings of the 55th Annual Meeting of the Association for Computational Linguistics (Volume 1: Long Papers), pages 451-462, Vancouver, Canada. Association for Computational Linguistics.

Mikel Artetxe, Gorka Labaka, and Eneko Agirre. 2018a. Generalizing and improving bilingual word embedding mappings with a multi-step framework of linear transformations. In Proceedings of the Thirty-Second AAAI Conference on Artificial Intelligence (AAAI-18), pages 5012-5019.

Mikel Artetxe, Gorka Labaka, and Eneko Agirre. 2018b. A robust self-learning method for fully unsupervised cross-lingual mappings of word embeddings. In Proceedings of the 56th Annual Meeting of the Association for Computational Linguistics (Volume 1: Long Papers), pages 789-798. Association for Computational Linguistics.

Alexis Conneau, Guillaume Lample, Marc'Aurelio Ranzato, Ludovic Denoyer, and Hervé Jégou. 2018. Word translation without parallel data. In Proceedings of the 6th International Conference on Learning Representations (ICLR 2018).

Georgiana Dinu, Angeliki Lazaridou, and Marco Baroni. 2015. Improving zero-shot learning by mitigating the hubness problem. In Proceedings of the 3rd International Conference on Learning Representations (ICLR 2015), workshop track.

Long Duong, Hiroshi Kanayama, Tengfei Ma, Steven Bird, and Trevor Cohn. 2016. Learning crosslingual word embeddings without bilingual corpora. In Proceedings of the 2016 Conference on Empirical Methods in Natural Language Processing, pages 12851295, Austin, Texas. Association for Computational Linguistics.

Chris Dyer, Victor Chahuneau, and Noah A. Smith. 2013. A simple, fast, and effective reparameterization of ibm model 2. In Proceedings of the 2013 Conference of the North American Chapter of the Association for Computational Linguistics: Human
Language Technologies, pages 644-648, Atlanta, Georgia. Association for Computational Linguistics.

Stephan Gouws, Yoshua Bengio, and Greg Corrado. 2015. BilBOWA: Fast bilingual distributed representations without word alignments. In Proceedings of the 32nd International Conference on Machine Learning, pages 748-756.

Yedid Hoshen and Lior Wolf. 2018. Non-adversarial unsupervised word translation. In Proceedings of the 2018 Conference on Empirical Methods in Natural Language Processing, pages 469-478, Brussels, Belgium. Association for Computational Linguistics.

Thang Luong, Hieu Pham, and Christopher D. Manning. 2015. Bilingual word representations with monolingual quality in mind. In Proceedings of the 1st Workshop on Vector Space Modeling for Natural Language Processing, pages 151-159. Association for Computational Linguistics.

Tomas Mikolov, Quoc V Le, and Ilya Sutskever. 2013a. Exploiting similarities among languages for machine translation. arXiv preprint arXiv:1309.4168.

Tomas Mikolov, Ilya Sutskever, Kai Chen, Greg S Corrado, and Jeff Dean. 2013b. Distributed representations of words and phrases and their compositionality. In Advances in Neural Information Processing Systems 26, pages 3111-3119.

Ndapa Nakashole and Raphael Flauger. 2018. Characterizing departures from linearity in word translation. In Proceedings of the 56th Annual Meeting of the Association for Computational Linguistics (Volume 2: Short Papers), pages 221-227, Melbourne, Australia. Association for Computational Linguistics.

Barun Patra, Joel Ruben Antony Moniz, Sarthak Garg, Matthew R Gormley, and Graham Neubig. 2019. BLISS in non-isometric embedding spaces.

Miloš Radovanović, Alexandros Nanopoulos, and Mirjana Ivanović. 2010a. Hubs in space: Popular nearest neighbors in high-dimensional data. Journal of Machine Learning Research, 11(Sep):2487-2531.

Milos Radovanović, Alexandros Nanopoulos, and Mirjana Ivanović. 2010b. On the existence of obstinate results in vector space models. In Proceedings of the 33rd international ACM SIGIR conference on Research and development in information retrieval, pages 186-193. ACM.

Sebastian Ruder, Ivan Vulić, and Anders Søgaard. 2017. A survey of cross-lingual word embedding models. arXiv preprint arXiv:1706.04902.

Víctor M. Sánchez-Cartagena, Marta Bañón, Sergio Ortiz Rojas, and Gema Ramírez. 2018. Prompsit's submission to wmt 2018 parallel corpus filtering shared task. In Proceedings of the Third Conference on Machine Translation: Shared Task Papers, 
pages 955-962. Association for Computational Linguistics.

Anders Søgaard, Sebastian Ruder, and Ivan Vulić. 2018. On the limitations of unsupervised bilingual dictionary induction. In Proceedings of the 56th Annual Meeting of the Association for Computational Linguistics (Volume 1: Long Papers), pages 778788. Association for Computational Linguistics.

Ivan Vulić and Marie-Francine Moens. 2016. Bilingual distributed word representations from documentaligned comparable data. Journal of Artificial Intelligence Research, 55(1):953-994.

Meng Zhang, Yang Liu, Huanbo Luan, and Maosong Sun. 2017. Adversarial training for unsupervised bilingual lexicon induction. In Proceedings of the 55th Annual Meeting of the Association for Computational Linguistics (Volume 1: Long Papers), pages 1959-1970, Vancouver, Canada. Association for Computational Linguistics. 